\title{
Calculating Apportionment of Metals in PM2.5 using Ni Isotope Characterization
}

\author{
M. Kelvin ${ }^{1,2 *}$, A. Voinot ${ }^{2}$, M. Leybourne ${ }^{2}$, D. Layton- \\ Matthews $^{2}$, S. Verpaele ${ }^{3}$ \\ (*Correspondence: michelle.kelvin@xps.ca) \\ ${ }^{1}$ XPS - Glencore, Sudbury, Ontario, Canada \\ ${ }^{2}$ Queen's University, Kingston, Ontario, Canada \\ ${ }^{3}$ Nickel Institute, Brussels-Belgium
}

Due to its versatile physicochemical properties, $\mathrm{Ni}$ is a valuable commodity used in wide range of modern technologies. As demand rises, $\mathrm{Ni}$ processing operations are tasked with maximizing production while limiting emissions, owing to increasing evidence of epidemiological risk. Monitoring sources of particulate matter (PM) generated at $\mathrm{Ni}$ operations is essential for preventing dust emissions from entering the environment. Extensive speciation work has been completed for $\mathrm{PM}_{10}$ using methods of quantitative mineralogy, but analysis of $\mathrm{PM}_{2.5}$ remains challenging due to the fine and ultrafine particle sizes. Nickel isotope geochemistry may be a practical tool for assessing sources of $\mathrm{Ni}$ in $\mathrm{PM}_{2.5}$, as mass-dependent variations in composition have recently been shown to be distinct between geological systems, and because $\mathrm{Ni}$ is relatively depleted in ambient air. A double spike MC-ICP-MS method is being used to determine $\mathrm{Ni}$ isotope ratios in materials that are potential sources of dust at processing operations. An automated, column ion-exchange purification process (ESI Prepfast) has the ability to produce high-yield, matrix-free solutions that are sufficient for analysing particulate matter with $\mathrm{Ni}$ concentrations approaching background levels $\left(<0.001 \mu \mathrm{g} / \mathrm{m}^{3}\right)$. A source specific apportionment of $\mathrm{Ni}$ in $\mathrm{PM}_{2.5}$ can be calculated based on the $\mathrm{Ni}$ isotope ratios and absolute $\mathrm{Ni}$ concentration. Comparison of initial results to the mineralogy of the source materials and baseline $\mathrm{PM}_{10}$ shows excellent promise for employing this Ni isotope procedure for characterizing $\mathrm{PM}_{2.5}$ in larger, dust sampling campaigns designed to suppress fine emissions. 